\title{
A Novel Approach to Implement Fixed to Mobile Convergence in Mobile Adhoc Networks
}

\author{
Dr. P.K.Suri \\ Professor \& Head, Dept. of Computer Sc and Applications \\ Kurukshetra University \\ Kurukshetra, India \\ pksuritf25@yahoo.com
}

\author{
Sandeep Maan \\ Assist. Professor, Dept. of Computer Sc. \\ Govt. P.G. College \\ Gurgaon, India \\ sandeep.mann23@gmail.com
}

\begin{abstract}
Fixed to Mobile Convergence, FMC is one of the most celebrated applications of wireless networks, where a telephonic call from some fixed telephonic infrastructure is forwarded to a mobile device. Problem of extending the reach of fixed telephony over a mobile ad-hoc network working in license free ISM band has been eluding the research fraternity. Major hindrance to FMC implementation comes from very nature of mobile ad-hoc networks. Due to the dynamic nature and limited node range in mobile ad-hoc networks, it is very difficult to realize QoS dependent applications, like FMC, over them. In this work authors are proposing complete system architecture to implement fixed to mobile convergence in a mobile ad-hoc network. The mobile ad-hoc network in the problem can hold a number of telephonic calls simultaneously. The proposed system is then implemented using network simulator, ns2. The results obtained are then compared with the predefined standards for implementation of FMC.
\end{abstract}

Keywords-Mobile Ad-hoc Networks; Packet Telephony; Voice over Internet Protocol; Fixed to Mobile Convergence; Quality of Service

\section{INTRODUCTION}

Mobile ad-hoc networks, MANET, are the latest member of illustrious family of wireless networks. In mobile ad-hoc networks, a number of autonomous and mobile nodes communicate with each other by forming multi-hop connections and maintaining connectivity in decentralized manner. MANETs are different from other wireless networks in terms of their distinct characteristics like limited range, unreliable media, dynamic topology, limited energy etc. In mobile ad-hoc networks each node is a potent router and forwards the packet received from other nodes using some suitable routing algorithm. The reason behind the popularity of mobile ad-hoc networks is their small setup time and deployment cost. These networks have proved their worth in emergency like situations such as natural calamity, war time etc

The area of mobile ad-hoc networks has taken another leap forward with invention of device working in license free Industrial-Scientific-Military (ISM) band concentrated at 2.4
$\mathrm{GHz}$. Two notable technologies working in this band are $\mathrm{WiFi}$ and Bluetooth.

Fixed to Mobile Convergence, also referred as FMC[1]-[2] during this work, represents the process of extending reach of fixed telephony with the help of wireless techniques. One method of achieving this can be using wireless handsets and a single antenna near the wired end. The range covered by this technique cannot be large. To cover a large area one would require to purchase some frequency, which is a costly affair. During this work authors proposes a system where calls are forwarded through a mobile ad-hoc network working in ISM band. This mobile ad-hoc network under study, apart from extending the reach of fixed call, will also allow other users of the network to call each other simultaneously.

The underlying technique to be used for call forwarding will be voice over internet protocol, also termed as packet telephony during this work. In Packet Telephony, data belonging to the telephonic conversations is transported as IP packets and no permanent copper path is setup between source and destination. In order to offer real time service over the mobile ad-hoc network certain Quality of Service (QoS) parameters play vital role. These parameters include end to end delay, packet delivery ratio and throughput etc. Measures are required to keep the values of QoS parameters in the specified range. Quality of Service offered by the mobile adhoc networks depends on a number of variables like node speed, number of nodes, number of connections, area etc. Major deterrent to offering QoS based services, over the mobile ad-hoc networks, is basic nature of these networks. Due to the dynamic nature of mobile ad-hoc networks routes are also very vulnerable and re-routing is required frequently.

In this paper, we propose complete system architecture to implement FMC through a MANET carrying number of simultaneous telephonic conversations. The proposed methodology for implementing fixed to mobile convergence would be using wireless technology working in license free ISM band for deriving maximum befits out of the technique. The proposed architecture is simulated using ns2 and the results are verified with practically acceptable values (table 1 ). 


\section{RELATED WORK}

Paolo Giacomazzi et al. [3] proposed architecture for mobile ad-hoc networks carrying VoIP based telephonic conversation. The authors evaluated their architecture in terms

TABLE I. QOS PARAMETERS

\begin{tabular}{|l|c|}
\hline $\begin{array}{c}\text { Acceptable Range for QoS Parameters to } \\
\text { successfully implement Packet Telephony }\end{array}$ \\
\hline $\begin{array}{c}\text { Critical QoS } \\
\text { Parameter }\end{array}$ & $\begin{array}{c}\text { Acceptable } \\
\text { Range }\end{array}$ \\
\hline End to End Delay & $<=120 \mathrm{~ms}$ \\
\hline Packet Delivery Rate & $>=95 \%$ \\
\hline Packet Drop Rate & $<=5 \%$ \\
\hline Packet Loss Rate & $<=5 \%$ \\
\hline
\end{tabular}

of various Quality of Service parameters like Call Drop Rate, MOS etc. The authors observed that the ad-hoc networks can be used for economically extending access of fixed infrastructure, termed as Fixed to Mobile Convergence (FMC). They also observed that although the size of this extension is quite limited.

\section{SYSTEM ARCHITECTURE}

The problem environment is sketched in figure 1. The system consists of a single fixed telephone and a number of mobile nodes forming a mobile ad-hoc network. The system not only forwards the calls at fixed telephone but various mobile nodes can themselves be engaged in telephonic conversations.

To start with, in packet telephony, analog voice conversations are to be converted to digital and then compressed. To cater the scarcity of available bandwidth with mobile ad-hoc networks, compression technique is to be carefully selected. Then the digital voice calls are converted into IP packets and forwarded over the mobile ad-hoc network. Finally, these packets are collected at destination; the digital voice is extracted from these and converted into analog voice.

During this work the authors have assumed that the area covered by the network is square in shape. As source and destination may not be neighbor to each other, the call forwarding can be multi-hop. Overall the proposed system architecture consists of five network layers [4]-[5] as explained below:

\section{A. Physical Layer}

The purpose of physical layer is to transmit the data from one node to other and hence from source to destination. A number of Physical Layer solutions for mobile ad-hoc networks are available like IEEE 802.11 PHY[6]-[9], UTRATDD [10]-[11]. The authors have proposed to used the most practically used solution in IEEE 802.11 based Simple Wireless Channel working in ISM band around $2.4 \mathrm{GHz}$.

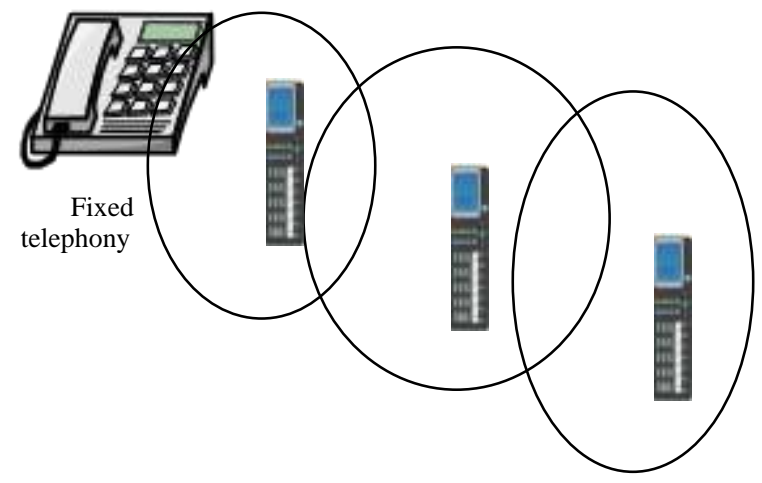

Figure 1. Fixed to Mobile Convergence over Mobile ad-hoc network

\section{B. MAC Layer}

For networks with small bandwidth at their disposal MAC layer is very critical. This layer has great impact on the performance of the Networks. There are number of MAC layer solutions like IEEE 802.11, VMAC etc. A good survey of popular MAC protocols can be found in [12]-[16]. The authors have used IEEE 802.11based MAC layer.

\section{Network Layer}

The major role played by the network layer is routing. A number of routing algorithms have been proposed. These algorithms have been classified into two categories [17] viz. topology based and position based routing [18]-[24] algorithms. Out of these most practical system employ topology based routing algorithms. Topology based routing algorithms have been further classified into three categories viz. proactive, reactive and hybrid. The authors have proposed to use Dynamic Source Routing, DSR [25] algorithm in this work.

\section{Transport Layer}

Due to the wireless nature of network UDP is used. To realize a quality of service based application like VoIP, one cannot rely completely on unreliable UDP rather Real-Time Transport Protocol (RTP) is run on top of UDP to provide end-to-end delivery services like payload type identification, sequence numbering etc.

\section{E. Application Layer}

This layer converts telephonic conversations into the digitized form. International Telecommunication Union has standardized a number of effective speech compression algorithms; In our scenario as overall traffic load is high as compared to the available bandwidth, authors are using G .729 codec [26] working at $8 \mathrm{kbps}(50$ packets/sec with a packet of size $20 \mathrm{~B}$. 
System proposed by the authors is sketched in figure 2 .

\section{Simulation}

Authors simulated the overall system in network simulator (ns2 ver 2.34) [27]. The system was implemented on Fedora Linux running on a Pentium computer. Number of simulation runs was made with different environmental settings. The performance of system was evaluated in terms of various Quality of Service parameters as given below:

\begin{tabular}{|l|}
\hline $\begin{array}{l}\text { G .729 codec at 8kbps } \\
\text { (Application Layer) }\end{array}$ \\
\hline $\begin{array}{l}\text { RTP/UDP } \\
\text { (Transport Layer) }\end{array}$ \\
\hline $\begin{array}{l}\text { Dynamic Source Routing } \\
\text { (Network Layer) }\end{array}$ \\
\hline $\begin{array}{l}\text { LLC/IEEE 802.11 MAC } \\
\text { (Data Link Layer) }\end{array}$ \\
\hline $\begin{array}{l}\text { IEEE 802.11 PHY } \\
\text { (Physical Layer) }\end{array}$ \\
\hline
\end{tabular}

Figure 2. Proposed System Architecture

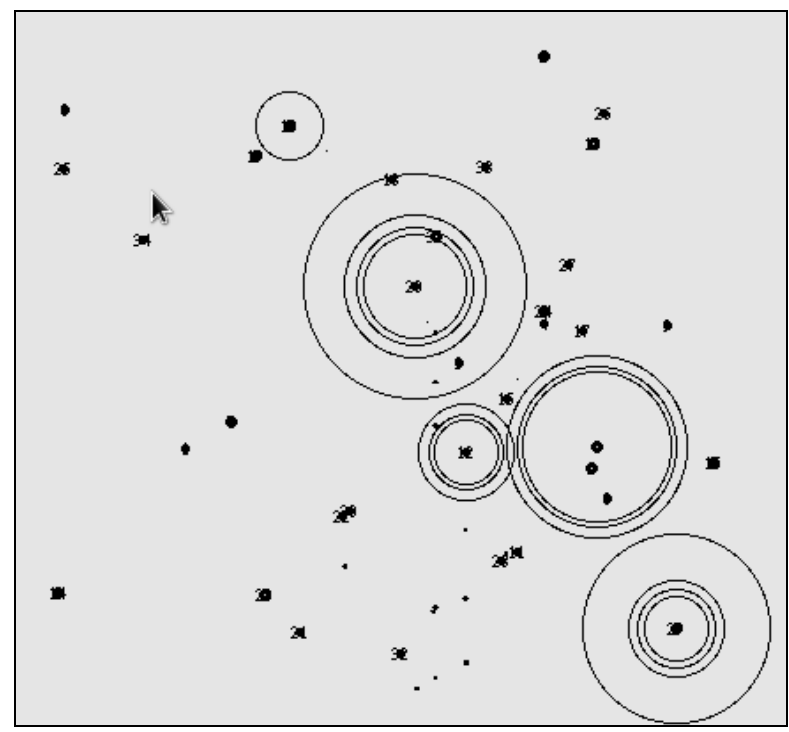

Figure 3. Snapshot of NAM trace

\section{A. Packet Delivery Ratio}

Packet delivery ratio signifies the percentage of packets that were successfully delivered from source to destination.
Failure to deliver a packet would mean loss of conversation data and hence incomplete conversation. So, a packet delivery ratio of more than $95 \%$ must be achieved for successful implementation of network.

\section{B. End to End Delay}

End to End delay represents the time elapsed between delivering of voice from speaker to listener. In quantitative terms a delay of $120 \mathrm{~ms}$ or less is acceptable. The problem comes if the delay is more than $250 \mathrm{~ms}$ as it ultimately leads to the half duplex conversation.

\section{Throughput}

Throughput represents number of successful packet transmissions per second. Throughput is very important evaluation parameter.

\section{Packet Drop Rate}

Packet Drop Rate represents percentage of packets that were dropped by intermediate nodes due to overloaded queue. Packet drop rate is significant as it identifies congestion in the network and data handling capability of a network. It is assumed that a mobile ad-hoc network would be unacceptable if packet drop rate goes beyond 5\%. More drop rate means more retransmission and hence more delays.

\section{E. Packet Loss Rate}

Packet loss rate represents percentage of packets that are lost into the network. This definitely means loss of conversation and hence a packet loss rate above $5 \%$ is highly unacceptable.

\section{RESULTS \& OBSERVATIONS}

The important observations are plotted below:

\section{A. Parameter: End to End Delay}

\section{Observations (figures 4-6):}

Authors observed that end to end delay increases with number of active connections but overall delay was with in the specified experimental limits. While with increased number of participating nodes delay decreases. Both results are on expected line as with increase in number of connections traffic load on the network increases thereby increasing chaces of congestion and hence delay. Whereas with increased number of participating nodes there will be lesser chances of route error and more than one route may exist simultaneously in route cache. 


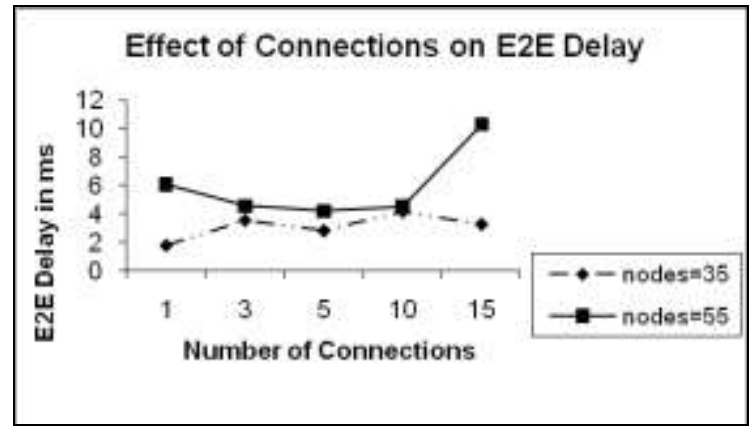

Figure 4. Effect of number of connections on End to End Delay (in ms) Other parameters are Area $=2.25 \mathrm{KM}^{2}$, Node Speed $=25 \mathrm{KMPH} \&$ Nodes $=35 / 50$.

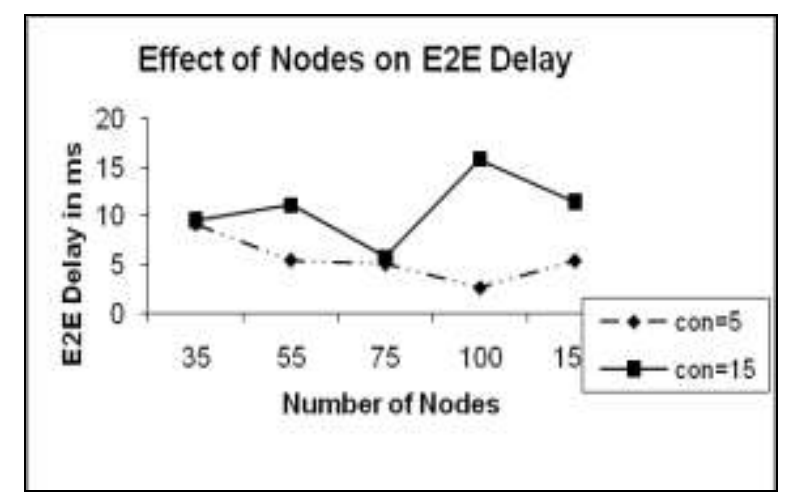

Figure 5. Effect of number of nodes on End to End Delay (in ms). The other parameters are Area $=2.25 \mathrm{KM}^{2}$, Node Speed $=25 \mathrm{KMPH} \&$ Connections $=5 / 15$.

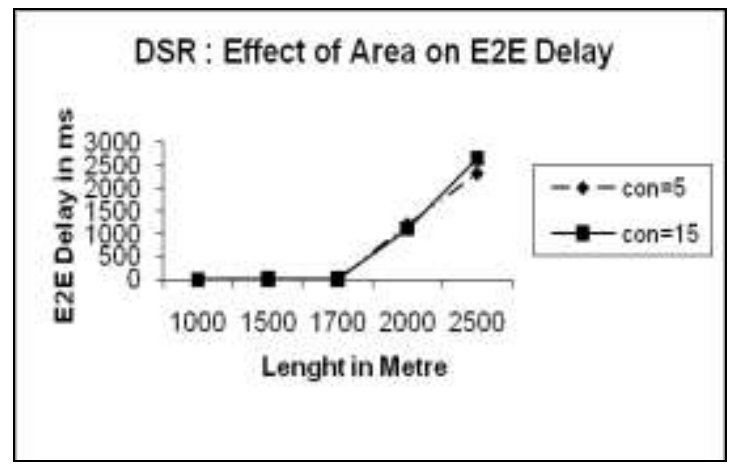

Figure 6. Effect of area (Area assumed to be square= Length* Length) on End to End Delay (in ms).

Important observation comes from the plot of E2E delay vs. area, it was observed that the delay was with in the allowable limits upto smaller network size, but for larger area E2E delay increases drastically and system performance becomes unacceptable.

\section{B. Parameter: Packet Delivery Ratio(PDR)}

Observations (figures 7-9):
Authors observed that packet delivery ratio (PDR) decreases with number of active connections but overall PDR was with in the experimental limits. With increasing number of participating nodes PDR remains almost constant. The result is as per expectations as with increase in number of connections traffic load on the network increases thereby increasing chaces of congestion and hence lesser PDR.

Again, important observation comes from the plot of PDR vs. area; it was observed that the PDR drops drastically for large network sizes.

\section{Parameter: Throughput \\ Observations (figures 10-12):}

Authors observed that throughput increases with number of active connections. While with increase in number of participating nodes, throughput stays almost constant. The result is as per expectations, because with increase in number of connections network has more packets to deliver and hence better throughput. It was also observed that the throughput decreases with increase in size of the network.

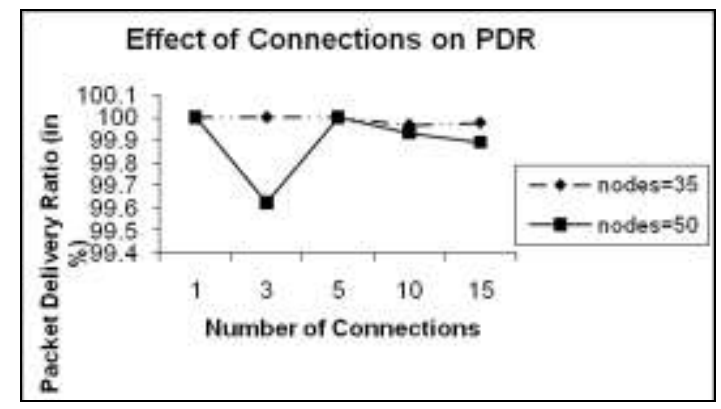

Figure 7. Effect of Number of Connections on PDR. The other parameters are Area $=2.25 \mathrm{KM}^{2}$, Node Speed $=25 \mathrm{KMPH} \&$ Nodes $=35 / 50$.

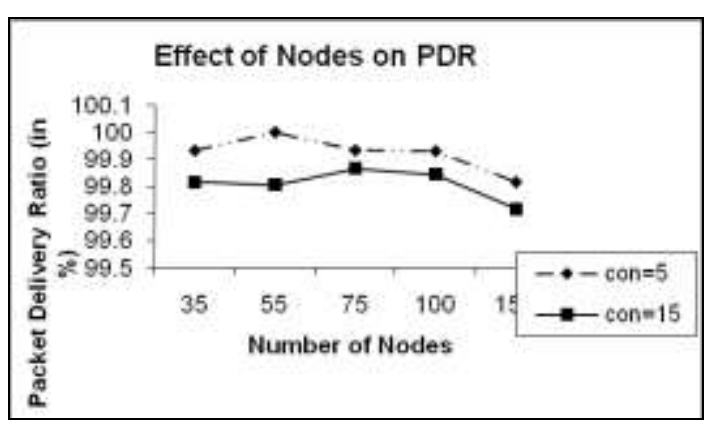

Figure 8. Effect of Number of Nodes on PDR. The other parameters are Area $=2.25 \mathrm{KM}^{2}$, Node Speed $=25 \mathrm{KMPH} \&$ Connections $=5 / 15$. 


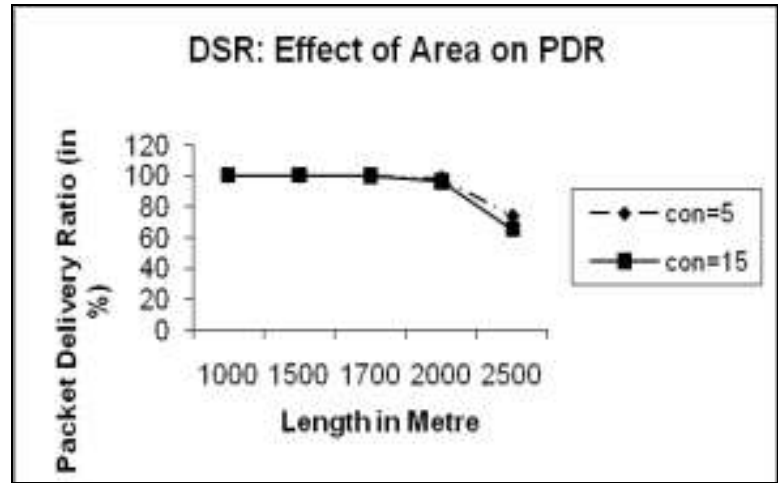

Figure 9. Effect of area (Area assumed to be square= Length* Length) on PDR.

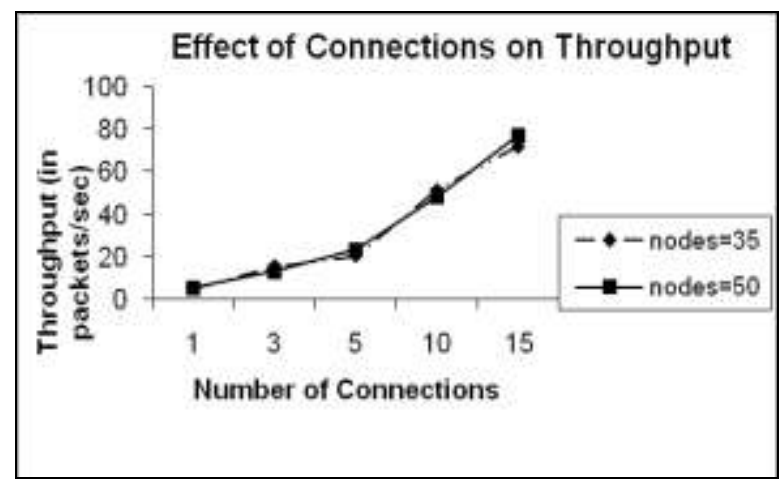

Figure 10. Effect of Number of Connections on Throughput for. The other parameters are Area $=2.25 \mathrm{KM}^{2}$, Node Speed $=25 \mathrm{KMPH} \&$

$$
\text { Nodes }=35 / 50
$$

\section{Parameter: Packet Drop Ratio(PDrR)}

\section{Observations (figures 12-15):}

Authors observed that Packet Drop Ratio remains in the experimental limits when plotted against number of nodes and number of connections. But it crosses allowable limit when the size of network increases.

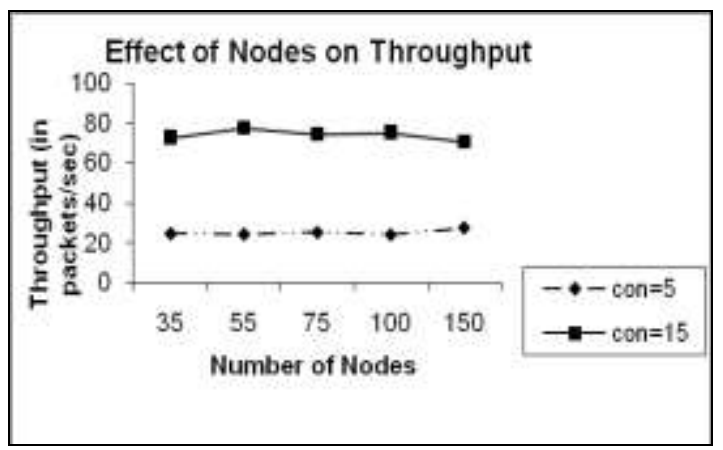

Figure 11. Effect of Number of Nodes on throughput. The other parameters are Area $=2.25 \mathrm{KM}^{2}$, Node Speed $=25 \mathrm{KMPH} \&$ Connections $=$ $5 / 15$.

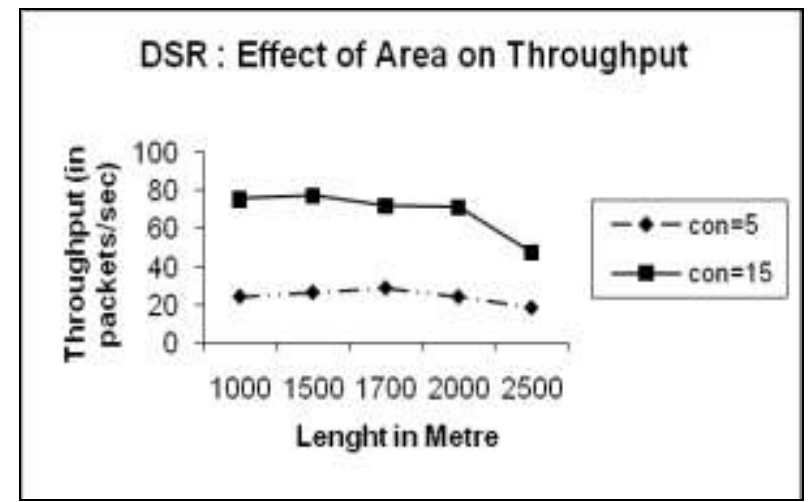

Figure 12. Effect of area (Area assumed to be square= Length* Length) on Throughput.

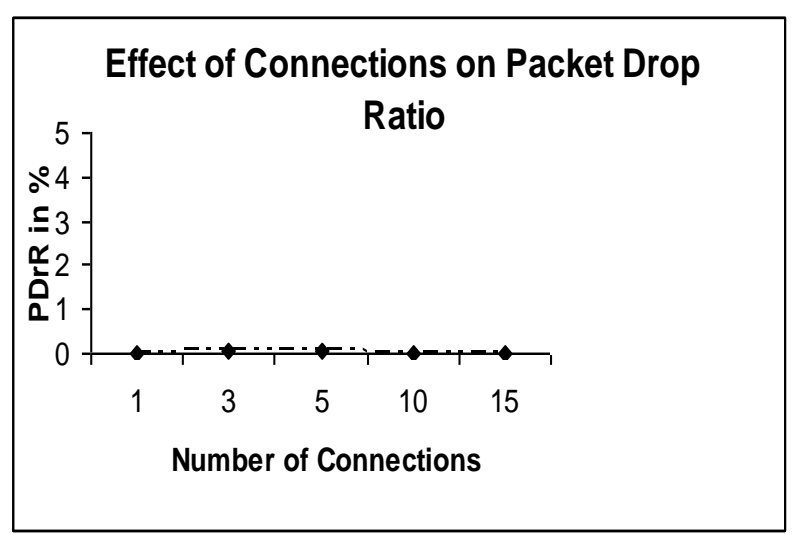

Figure 13. Effect of Number of Connections on Packet Drop Ratio for Area $=2.25 \mathrm{KM}^{2}$, Node Speed $=25 \mathrm{KMPH} \&$ Nodes $=35$

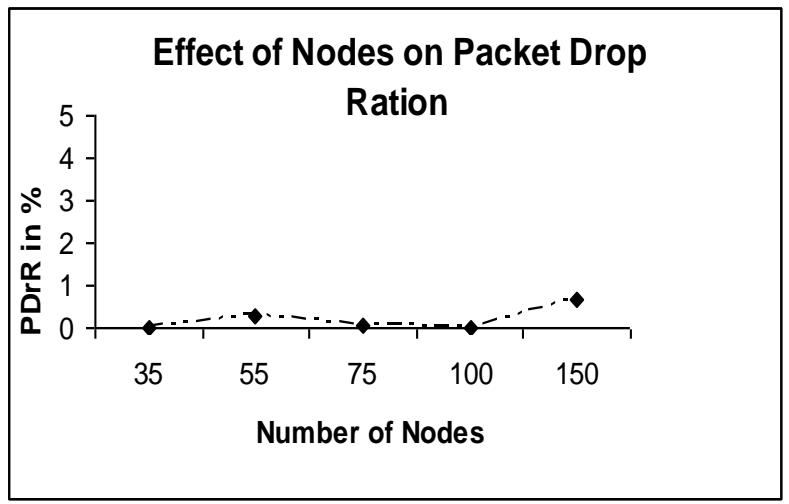

Figure 14. Effect of Number of Nodes on Packet Drop Ratio. The other parameters are Area $=2.25 \mathrm{KM}^{2}$, Node Speed $=25 \mathrm{KMPH} \&$ Connections $=5$.

\section{CONCLUSION}

In this work we presented complete system architecture for implementing fixed to mobile convergence using a mobile adhoc network. Fixed to mobile convergence can prove to be very useful if implemented using mobile ad-hoc networks. It 


\section{Effect of Area on Packet Drop Ratio}

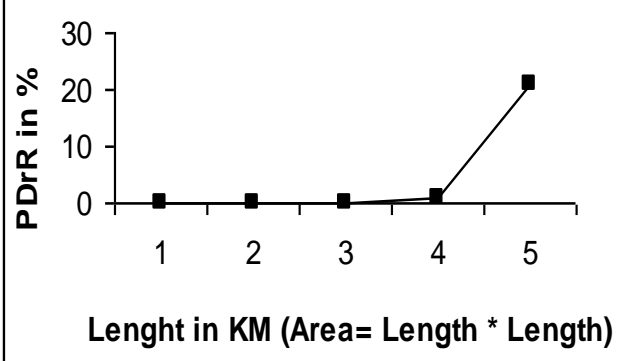

Figure 15. Effect of area (Area assumed to be square= Length* Length) on Packet Drop Ratio.

would provide a cost effective and fast solution to the problem of extending reach of existing fixed telephony. For this purpose network architecture was proposed during this work. The proposed architecture was then simulated using ns 2 and observed results were compared with proposed practical limits for various network parameters like End to End Delay, Packet Delivery Ratio and throughput.

It was observed that it is possible to successfully implement FMC using a mobile ad-hoc network but this extension cannot cover a large area, it was observed that it was possible to cover a network size of around $3 \mathrm{KM}^{2}$ with the proposed system architecture.

\section{FUTURE SCOPE}

During this work the feasibility of fixed to mobile convergence using a mobile ad-hoc network was evaluated using network layer parameters. Other authors are encouraged to evaluate perfprmance of proposed architecture under different MAC level protocols.

\section{REFERENCES}

[1] J. Vong J. Srivastava, and M. Finger, "Fixed to Mobile Convergence (FMC): technological convergence and the restructuring of the European telecommunications industry", SPRU 40th Anniversary B12Conference, 2006.

[2] L. Combat et. al., "Tactical Voice over Internet Protocol (VOIP) in secure wireless networks", June 2003.

[3] Paolo Giacomazzi, Luigi Musumeci, Giuseppe Caizzone, Giacomo Verticale, G. Liggieri, A. Proietti and S. Sabatini, "Quality of Service for Packet Telephony over Mobile Ad Hoc Network", IEEE Network, Jan/Feb 2006.

[4] J.Schiller, "Mobile Communications", Pearson Education, Third Indian Reprint, 2004

[5] Andrew S. Tanenbaum, "Computer Networks", PHI.

[6] F. Cali, M. Conti, and E. Gregori. "IEEE 802.11 Wireless LAN: Capacity Analysis and Protocol Enhancement," IEEE INFOCOM 1998, vol. 1, pp. 142-49.

[7] "Information Technology-Telecommunications and Information Exchange Between Systems - Local and Metropolitan Area NetworksSpecific Requirements - Part 11: Wireless LAN Medium Access
Control (MAC) and Physical Layer (PHY) Specifications", IEEE Std 802.11-1997.

[8] D.J. Deng et. al., "A priority scheme for IEEE 802.11 DCF access method," IEICE Trans. Commun., 1999, pp 96-102.

[9] G. Bianchi. "Performance Analysis of the IEEE 802.11 Distributed Coordination Function," IEEE JSAC, vol. 18, no. 3, Mar. 2000, pp. 53547.-U

[10] M. Lott et al., "Medium Access and Radio Resource Management for Ad Hoc Networks Based on UTRA-TDD," Proc. 2nd ACM Int'l. Symp. Mobile Ad Hoc Net and Comp., 2001.

[11] A. Ebner et al., "Performance of UTRA TDD Ad Hoc and IEEE 802.11b in Vehicular Environments," Proc. IEEE VTC 2003-Spring, vol. 2, Apr. 2003, pp. 18-22.

[12] Sunil Kumar, Vineet S. Raghavan, Jing Deng, "Medium Access control protocols for ad hoc wireless networks: A survey", Ad Hoc Networks, 2006, pp 326-358.

[13] A. Pal et al., "MAC layer protocols for real-traffic in ad hoc networks," Proceedings of the IEEE International Conference on Parallel Processing, 2002.

[14] A. Muir et. al., "An efficient packet sensing MAC protocol for wireless networks," Mobile Networks, 1998, pp 221-234.

[15] A. Nasipuri et. al., "A multichannel CSMA MAC protocol for multihop wireless networks," IEEE WCNC, September, 1999.

[16] C.R. Lin et. al., "MACA/PR: An asynchronous multimedia multihop wireless network," Proceedings of the IEEE INFOCOM, March 1997.

[17] E. M. Royer and C.-K. Toh. "A Review of Current Routing Protocols for Ad Hoc Mobile Wireless Networks," IEEE Pers. Commun., vol. 6, no. 2, Apr. 1999.

[18] T. Camp, J. Boleng, and L. Wilcox, "Location Information Services in Mobile Ad Hoc Networks," Proc. IEEE ICC, Apr. 2002, pp. 18-22.

[19] B. Karp et. al. "Greedy perimeter statelessrouting for wireless networks.", Proc. of the 6th Annual ACM/IEEE Int. Conf. on Mobile Computing and Networking (MobiCom 2000), pp 243.

[20] B. N. Karp, "Geographic Routing for Wireless Networks.", PhD thesis, Harvard University, 2000.

[21] C. E. Perkins and E. M. Royer, "Ad-hoc On-Demand Distance Vector Routing.", Proc. 2nd IEEE Workshop Mobile Comp. Sys. and Apps., Feb. 1999 , pp. 90-100.

[22] C. K. Toh, "A Novel Distributed Routing Protocol To Support Ad Hoc Mobile Computing," Proc. 1996 IEEE 15th Annual Int'1, pp. 480-86.

[23] C.C. Chiang, "Routing in Clustered Multihop, Mobile Wireless Networks with Fading Channel.”, Proc. IEEE SICON '97, Apr. 1997, pp. 197-211.

[24] C.E.Perkins et. al., "Highly Dynamic Destination Sequenced DistanceVector Routing (DSDV) for Mobile Computers.", Comp. Commun.Rev., Oct. 1994, pp. 234-44.

[25] D.B. Johnson and D.A. Maltz, "Dynamic Source Routing in Ad Hoc Wireless Networks," Mobile Computing, pp. 153-81.

[26] M. E. Perkins et al., "Characterizing the Subjective Performance of the ITU-T $8 \mathrm{~kb} / \mathrm{s}$ Speech Coding Algorithm ITU-T G.729," IEEE Commun. Mag., vol. 35, no. 9, Sep. 1997 pp. 74-81.

[27] Stuart Kurkowski, Tracy Camp and Michael Colagrosso, "MANET Simulation Studies: The incredible", Mobile Computing and Communication Review, vol 9, no 4, 2005.

\section{AUTHORS PROFILE}

Dr. P.K.Suri (pksuritf25@yahoo.com) has been working in the department of Computer Science and Applications, Kurukshetra University, Kurukshetra, India for more than twenty five years. He has guided a number of $\mathrm{PhD}$ students. His areas of specialization includes Computer based simulation and modeling, Computer Networks etc. Presently he is acting as Head Computer Sc department and Dean Faculty of Science \& Engineering in the university. 
Sandeep Maan (sandeep.mann23@gmail.com) is presently working as Assistant Professor in Computer Science at Govt. Post Graduate College, Sector-14, Gurgaon, India. He completed his M.Tech in Computer Science and Engineering from Kurukshetra University Kurukshetra. He is presently pursuing his $\mathrm{PhD}$ in Computer Science from Department of Computer Sceince and Applications,Kurukshetra University, Kurukshetra. His areas of interest includes Mobile Adhoc Networks, System Simulations and Artificial Intelligence 